\title{
Intrahepatic Biliary Atresia
}

National Cancer Institute

\section{Source}

National Cancer Institute. Intrahepatic Biliary Atresia. NCI Thesaurus. Code C97070.

A congenital disorder characterized by blockage or absence of the intrahepatic bile ducts. 